\title{
Using 4G Elements to Provide Secure Services Network Access
}

\author{
Cristina-Elena Vintilă \\ Bucharest, Romania, \\ Military Technical Academy
}

\begin{abstract}
The entire telecommunications and services world has focused its attention on the newest $3 G P P$ architecture, 4G. Proposing high speed data transfer, all flat-IP network for the core systems and stronger authentication and $A A A$ methods, this architecture seems to be the answer for the mobile networks of the future. Another architecture, described by $3 G P P$ as well, but based on IETF standards is the IMS architecture. This is focused on mobile services, mainly VoIP, but also presence, push-to-talk, location and multimedia and broadcast services. The IMS core network has its own security mechanisms, which are constantly tested and improved. Looking in the perspective, the user equipment, specially the one found in roaming has to do a lot of signaling in order to authenticate to both networks and use the services available for it in a secure manner. From the provider's point of view, the issue is even more complicated, because it has to secure its network, securely interoperate with other networks, be able to obtain granular charging information and also provide lawful interception capabilities for the government institutions. This paper reviews the authentication security architecture available in $3 G P P$ for providing secure access to application services, with emphasis on the interaction of $4 G$ and IMS components, being the first step in a larger $4 G$ IMS interaction study with regards to security.
\end{abstract}

\section{Introduction}

The 3GPP project consists of major telecommunications organizations worldwide and one of the latest architectures described by this organization is $4 \mathrm{G}$ - SAE - System Architecture Evolution. SAE consists of a radio network, named LTE - Long Term Evolution and a core network, named EPC - Evolved Packet Core, a network architecture based only on IP addressing. The radio network is out of the scope of this paper, the focus being on the components located in EPC, also referred to as EPS - Evolved Packet System. Another architectural design created by 3 GPP is IMS - IP Multimedia Subsystem, dealing initially with VoIP signaling, and later on with multiple types of services and applications, like push-to-talk, presence and MBMS. The IMS was introduced by 3GPP on Release 5 , as a core network services for the $3 \mathrm{G}-$
UMTS system. In time, it has evolved to include a large number of services, types of application servers, security enhancements and entities and enhancements that provide compatibility with other types of services networks. This paper presents the 4G-IMS architectures interaction, summarizing their main components. Then, the GAA - Generic Authentication Architecture system is described in more detail. The EPS-AKA and IMS-AKA procedures are presented, in order to have a view of the available alternatives when it comes to access control, via the access network, in this case the 4G network, versus the application network, in this case the IMS core.

The purpose of this paper is to propose a model of thinking that is no longer modular: so far, each architectural design focused on how to provide an allin-one system that would take care of everything. The security aspect was part of the model, so that the system should not rely on an outside security system. This approach is very good and normally assures the best level of security; in order to get to a system, the attacker would have to be able to pass by multiple layers of security factors. This paper comes up with the proposition of a lighter security design, where technologies that are security-enhanced on their own model can actually rely on different security architecture, not part of their design, and still be provided with a high security level. Although this model might not provide the best security solution, it has the advantage of being lighter in terms of computational weight and still provide a good security factor.

\section{Research Rationale}

The components most commonly found in EPC, along with their IMS interaction, are presented in the following figure. One of the main components of the EPC is the eNodeB, which has the functionality of the antenna and the controller. This component has the role of UE - User Equipment radio management, it is the first point of contact for the UE when connecting to a $4 \mathrm{G}$ network; it deals with the signaling for the UE management and also with the forwarding of the user-plane traffic to and from the UE. The signaling protocol specific to $4 \mathrm{G}$ is called GTPv2 or eGTP - evolved GTP. This protocol is present on the following EPC interfaces: S1-MME, $\mathrm{S} 11$ and S5/S8 and S4 (not presented in the picture, it 
appears between the SGSN and SGW). The userplane protocol present in EPC is GTPv1 - user plane, and it appears on the following interfaces: S1-U and $\mathrm{S} 5 / \mathrm{S} 8$. One or more eNodeBs (a pool of eNBs) is managed by an entity called MME - Mobility Management Entity. This device has the role of authenticating the UE to the HSS, it manages the UE sessions and controls its mobility over the network, and, unlike its homologous SGSN (Serving GPRS Support Node) device from $3 \mathrm{G}$, only has role in the signaling path of the EPC, no user-plane traffic flowing through it. The MME is the entity responsible with the selection of the following entity, SGW - Serving Gateway (it's homologous in 3G being the GGSN - Gateway GPRS Support Node).

The MME, eNB and UE entities are the three participants in the security context of a $4 \mathrm{G}$ security network design. In the $3 \mathrm{G}$ security design, only the user-plane traffic was being authenticated and confidentiality protected by keys negotiated between the UE and the AuC. The authentication and encryption keys (noted IK and respectively CK) were being kept on the SGSN. This model has several disadvantages that are being addressed by the 4G EPS key hierarchy, which determines that both the dataplane and the control-plane traffic are to be secured. The user/data-plane is authenticated and encrypted, just as in the $3 \mathrm{G}$, while the control-plane is also being secured. The key hierarchy is more complicated in 4G and involves a dedicated MME key used specifically for authenticating the NAS - Non-Access Stratum traffic, the control-plane traffic between the $\mathrm{UE}$ and the MME.

The SGW is the local mobility anchor for the UE: it manages the UE sessions and mobility, whether the UE is in active or in idle state, does QoS enforcement and forwards the control-plane and userplane messages to the next entity, the PGW - PDN (Packet Data Network) Gateway. The SGW is chosen by the MME according to specific criteria and it can be relocated. The decision whether to relocate or not the SGW belongs also to the MME. This entity verifies the presence of an S1-U interface between the current SGW and the target eNB. If this interface exists, it usually does not relocate the SGW. If this interface does not exist, it produces a list of SGW entities that have S1-U connection to the target eNB, and chooses the one that best matches all the rest of applicable criteria. The PGW entity has the role of allocating IP addresses to the UE, to route the traffic between the EPC and the PDN, to filter the traffic and assign it to different QoS classes, as well as to enforce this QoS for certain traffic. It is as well the mobility anchor for inter-working with non-3GPP technologies.

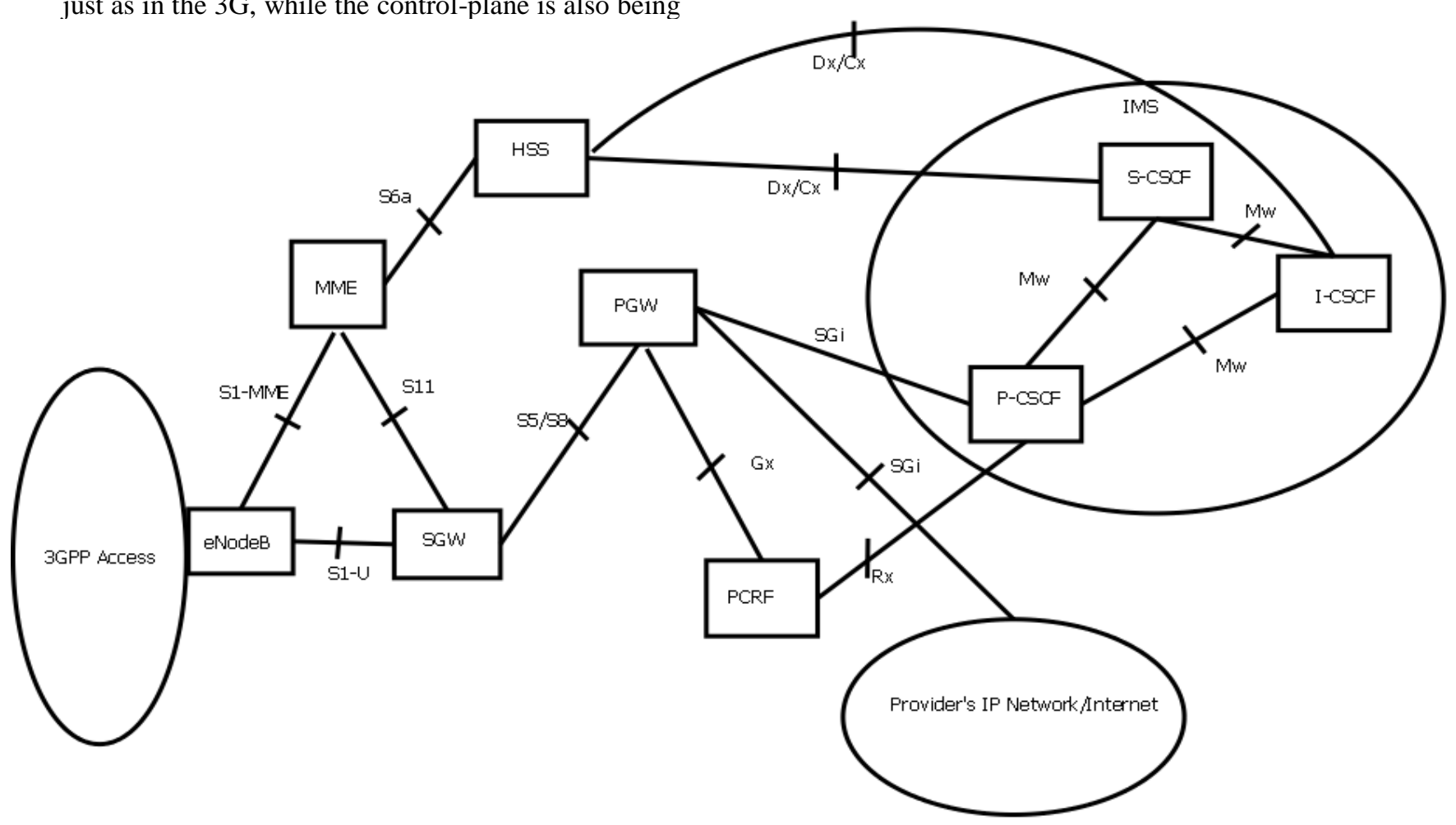

Figure 1. EPC - IMS Architecture

Another important aspect of the EPC is the QoS and the way it is enforced by the EPC elements. The traffic is assigned to different QoS classes based on the rules present in the PCRF. The HSS - Home Subscriber Server is a database system used to keep the SAE - related information about a certain UE, like the authentication information or the APN Access Point Name it can connect to. Unlike HSS, the PCRF - Policy Control and Rules Function contains the charging information about a certain user 
subscription, information based on which the PCEF Policy Control Enforcement Function component of the PGW provides QoS authorization (class identifiers and bitrates) and enforces this on the traffic routed through this device. In order to signal the creation of a QoS pattern for the traffic, the EPC uses the concept of bearers. A bearer is a data structure reserved on all the EPC components, it refers to the connection between a certain UE and an APN, for a certain traffic (identified by a TFT Traffic Flow Template - a set of TCP/IP port numbers and a QoS: QCI - QoS Class Identifier and a set of uplink and downlink bit rates).

The role of the bearer is to have independent classes of traffic granularly identified on the EPC components, situation useful when doing traffic shaping and for charging. One other important situation where these bearers are very useful is the handover process; the handover process is the process when an UE moves from the coverage area of an eNB to another eNB's area. The target antenna and the connected EPC components may or may not support the bitrates and bandwidth supported before the mobility took place. In the case where the target components no longer support the services the UE had before mobility, the EPC drops some of the bearers; the decision of what bearers to drop, meaning what services will no longer be available for that user is taken based on the bearers classification, created from a field called ARP - Allocation and Retention Priority: the bearers with the poorest ARP will be the ones dropped in a situation like the one described.

The PGW connects the UE to an APN via the SGi interface. This interface is a simple IP addressing network, but the APN can be an intranet, the Internet or an IMS - IP Multimedia Subsystem network. In case this is an IMS network, the PGW will most probably be connected to the P-CSCF entity of IMS. The center of the IMS is the CSCF - Call Session Control Function, functionality divided into three components: a Proxy - P-CSCF, an Interrogating unit - I-CSCF and a Serving unit - S-CSCF. The P is the first point of contact in the IMS network, whether the user is in the home network or in roaming; it is also the entity sitting in the signaling path, being able to do message inspection, can do compression of the SIP header (SigComp) and it is the one establishing IPSec sessions to the UE. If it includes a PDF - Policy Decision Function component, it can also do media-plane QoS enforcement and bandwidth management. The $\mathrm{S}$ is the central SIP server of the architecture, doing registrations, inspection of the messages (as it sits in the message path) and it decides the SIP AS Application Server which serves a certain service request. In its turn, the $S$ is assigned to the UE by the HSS. Being in the path of the messages, the $\mathrm{S}$ is also responsible for charging records generation. The I is another component located at the edge of the administration domain, where the other servers locate it by doing DNS interrogations (as it uses NAPTR, DNS and SRV records). It has the role of interrogating the HSS and finding out which $\mathrm{S}$ that HSS is allocating for that specific user. Just as the EPC, the IMS also relies on the existence of an HSS database, as well as a PCRF system. In case there are multiple IMS networks working together, and therefore multiple HSS databases present, a new element appears - SLF - Subscriber Location Function, which has the purpose of delivering a view from all the databases in order to find information about a user. The protocol dominant in IMS is SIP Session Initiation Protocol, standardized by IETF, having multiple extensions and improvements added to it. The protocol used to access the HSS is called Diameter, the more secure and more flexible follower of RADIUS protocol. The interfaces to HSS are all running Diameter, as well as the $\mathrm{P}$ interface to PCRF, Rx.

\section{GAA architecture}

As TR 33.919 [15] describes, the GAA - Generic Authentication Architecture is a mechanism created in order to address the need of certain applications for authentication. Initially created for application servers like presence, PKI portals or MBMS content server, this mechanism establishes a basis for any future mutual authentication needs of the applications, including the one described in this paper, IMS. The overall GAA architecture is composed of two main mechanisms: GBA - Generic Bootstrapping Authentication, described in TS 33.220 [13], which describes the usage of shared secrets in order to authenticate the users to the 4G systems, and SSC Support for Subscriber Certificates [16], which describes the procedures of using digital certificates by the user and the network in order to achieve mutual authentication when the mobile device accesses the network; this design requires the existence of a functional PKI infrastructure. Both these specifications describe ways to generate the authentication material and distribute it to the peers, being based on the AKA - Authentication and Key Agreement protocol or on an EAP - Extensible Authentication Protocol framework using the AKA method. The GBA architecture can be described, in a simple manner, as having six elements: HSS, BSF, NAF, ZnProxy, SLF and UE. The figure below presents a simplified network architecture for GBA when the Home Network and Visited Network are different network, separated by untrusted networks.

The HSS - Home Subscriber Server is the database system that stores the user's authentication credentials, generically named USS - User Security Settings (for example: GUSS - GBA User Security 
Settings), being the only entity that permanently stores these credentials. It has the purpose of mapping the GUSS to one or more private identities, but a private identity can only have at most one GUSS associated; a private identity in IMS is called IMPI IP Multimedia Private Identity

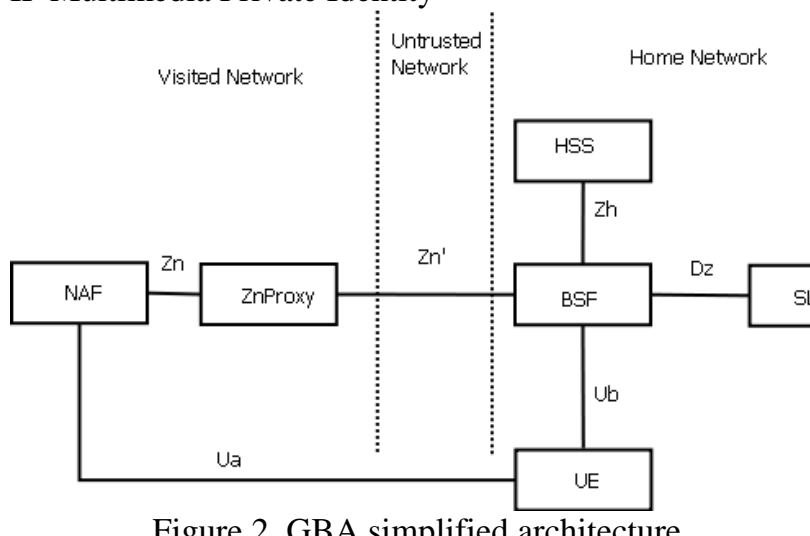

The GUSS format has many parameters, but the ones specifically needed here by the BSF are the following: the type of UICC used, the lifetime of the key generated for the subscriber and, optionally, the timestamp when the GUSS was modified in the HSS and they are sent by the HSS to the BSF via the $\mathrm{Zh}$ interface. The BSF - Bootstrapping Server Function is the entity that connects to the HSS via the $\mathrm{Zh}$ interface, in order to authenticate the user. The negotiation between the BSF and the UE is done via the Ub interface, and it runs the AKA protocol. The BSF connects to the HSS, retrieves the GUSS from the HSS, along with an AV - Authentication Vector which contains a pair of 5 values: RAND, AUTN, XRES, IK, CK. The BSF forwards RAND and AUTN to the UE. The UE - User Equipment has an UICC - Universal Integrated Circuit Card, which computes the IK, CK, MAC and RES based on the RAND and AUTN fields received from the BSF. The UE also verifies the MAC in order to authenticate the network, then it obtains the Ks by concatenating the IK and CK and sends the RES back to the BSF, in order to be authenticated by the network. The BSF verifies the RES against the XRES it has computed and, if they match, the UE is authenticated. If this happens, the BSF computes a Ks key, sends back to the UE a B-TID - Bootstrapping Transaction Identifier and the lifetime of the Ks. Both the UE and the BSF store this Ks and the B-TID for further use. The UE also stores the RAND value. The NAF Network Application Function is the generic server contacted by the UE. As per the Spec, there is no prior security association between the UE and the NAF. The BSF has the role to derive a Ks_NAF key and send it to the NAF via the $\mathrm{Zn}$ interface, while the UE also generates a Ks_NAF key. Of course, the BSF of that specific UE and the NAF this UE tries to connect to must have connectivity to each other, otherwise the key exchange will not be possible. Also, the NAF must have connectivity to the BSF of that UE, because it may need to access the HSS and get GUSS of the UE. The structure of the NAF is organized in policies: there are local policies that indicate whether a specific UE can have access or not to a certain application functionality. Also, in the application-specific USS there can be a key selection indication. When this happens, and if the NAF supports UICC-based enhancements for GBA (GBA_U), then this key selection indication overwrites the local policy of the NAF. The BSF should keep a list of NAFs and a list of groups of

${ }^{\text {SLI }}$ NAFs, in order to be able to identify at any given moment which NAF should be chosen if an application-specific USS appears. The definition of the NAFs in a group and the connection between these groups and the BSFs is in the implementation decision of the operator.

The ZnProxy entity appears in the architecture in the moment when the UE is in the visited network, the NAF is also operated by this visited network, but the BSF managing this UE is located in the home network. The ZnProxy may be a separate device, but it is usually in the functionality of an already existing device that has another primary role in the network: that of the visited NAF, the visited AAA server or an HTTP server - nevertheless, it must support Diameter and/or HTTP proxy functionality. The purpose of the ZnProxy in this architecture is to locate the UE's home BSF, to make sure it can securely connect to this device and to connect to it and send it the visited NAF DNS name. The SLF - Subscriber Locator Function is the entity queried by the BSF over the Dz interface to identify the HSS that contains the information about a specific UE. Just as with the IMS, this entity appears when there are multiple HSS devices. The bootstrapping procedure is described in the picture below. The UE includes the "3gpp-gbatmpi" token in the Request message it sends to the BSF over the Ub interface, then the BSF includes this token as well in the Response.

1. UE sends the HTTP request to the BSF, inserting an user identity, either its IMPI or its TMPI, if it has a temporary ID available;

2. The BSF identifies whether it received a TMPI or an IMPI; if it was a TMPI, it looks for the corresponding IMPI in its cache, and if it's not found, it gives an error to the UE, requesting the IMPI, otherwise it continues authenticating the UE to the HSS.

Then the BSF tries to locate the HSS and retrieve the GUSS and the AV from it, where $A V=($ RAND $\|$ AUTN $\|$ XRES $\|\mathrm{CK}\| \mathrm{IK})$, over Zh;

3. BSF forwards the RAND and AUTN to the UE, in order to authenticate it;

4. The UE uses AUTN to authenticate the network, then computes the XRES, CK and IK and 
5. sends the XRES to the BSF, in order to be authenticated by this entity and

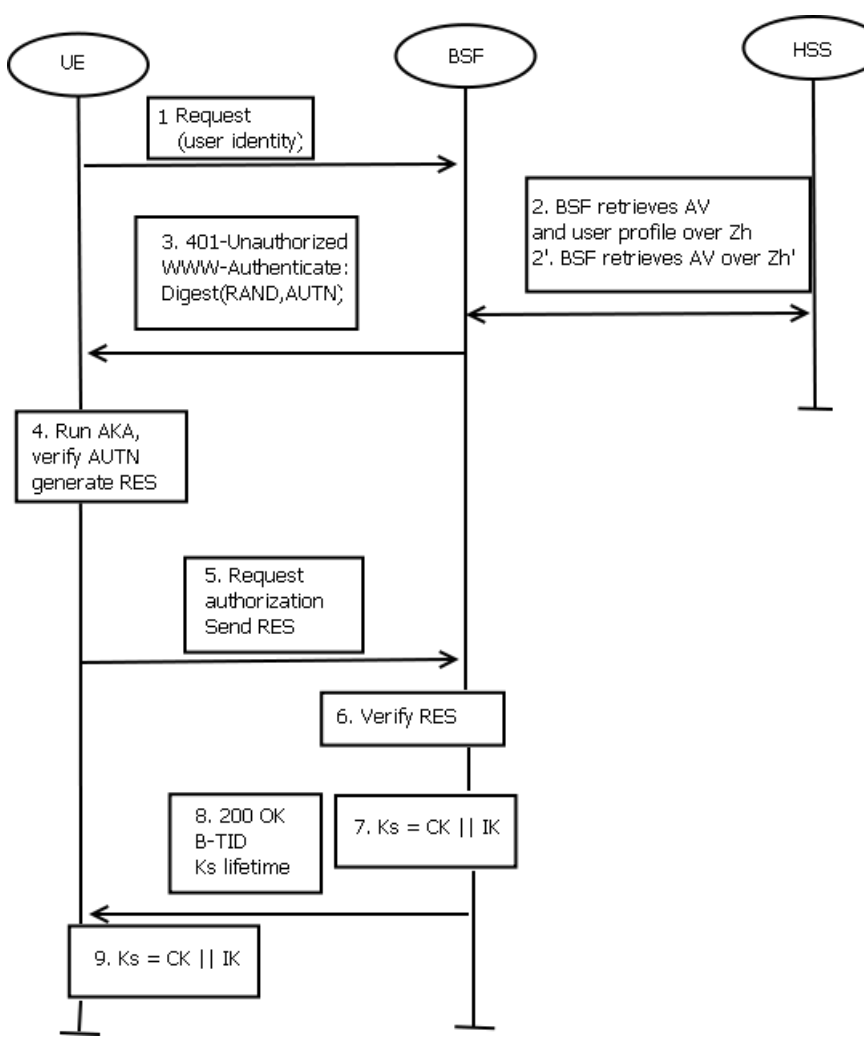

Figure 3. GBA procedure

6. the BSF verifies the XRES against its already computed RES; if they match, the UE is authenticated;

7. The BSF obtains the Ks by concatenating $\mathrm{CK}$ and IK, same as the UE and

8. replies to the UE with a B-TID in the 200 OK message;

9. The UE also obtains the Ks by concatenating its $\mathrm{CK}$ and IK

At this point, both the UE and the BSF derive the Ks_NAF key, the actual key that will be used to secure the communication between the $\mathrm{UE}$ and the NAF.

Ks_NAF $=\mathrm{KDF}(\mathrm{Ks}$, "gba-me", RAND, IMPI, NAF_Id), where KDF is the key derivation function and the NAF_Id looks like this: NAF_Id = FQDN of the NAF \| Ua security protocol identifier. All the values possible and structure of these components are defined in annexes to [13]

\section{An alternative: IMS AKA procedure}

When considering the $4 \mathrm{G}$ GAA design, the IMS network should play the role of the NAF server, more exactly, the P-CSCF component of the IMS, the contact point for the UE to its IMS network, should play the role of the NAF. This way, the entire GAA
AKA procedures should be performed as described so far, with the only clarification that the NAF server is the P-CSCF and that this particular device has is able to communicate with the home-network HSS.

Another approach for securing the $4 \mathrm{G}$ access to the IMS network is to use the SIP-AKA procedure for authenticating the SIP endpoint to the home-network HSS server, then secure the communication between the endpoint and the P-CSCF using the CK and IK keys derived by AKA, using IPSec.

The captures below are the four packets from the SIP-AKA negotiation between a SIP endpoint and a P-CSCF entity from OpenIMSCore [17]. There is only the SIP header displayed, as the rest of the packet is clear IP and UDP (or TCP) traffic.

The figure below presents the steps of the SIP Diameter negotiation between the elements involved in this process.

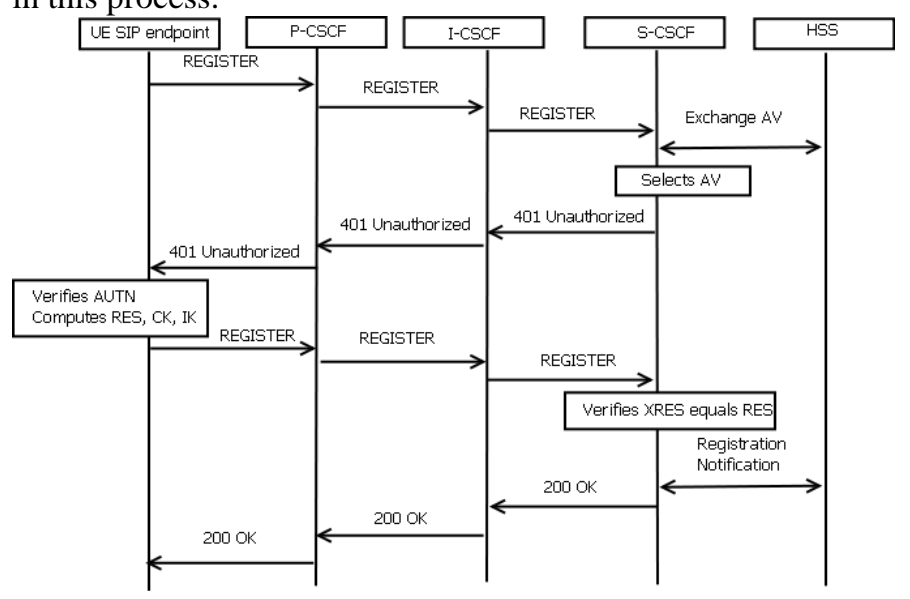

Figure 4. SIP-IMS-AKA authentication procedure

This first packet is the SIP REGISTER method sent from the UE to the P-CSCF; it does not contain any authentication methods and the details of each sub-header have been collapsed:

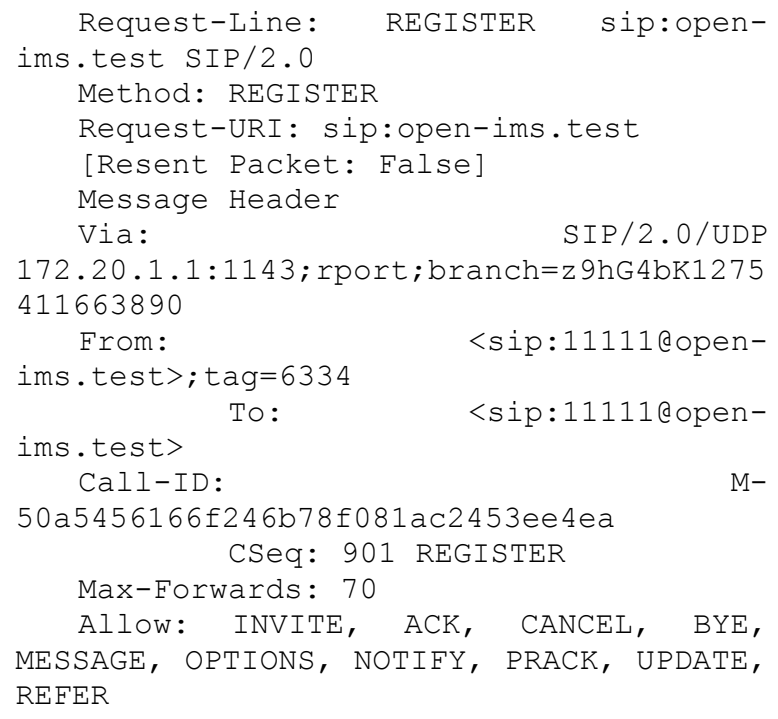


Contact:

<sip:11111@172.20.1.1:1143; transport=udp

$>$; expires $=600000$; +deviceID=" $3 \mathrm{ca} 50 \mathrm{bcb}$ -

7a67-44f1-afd0-

$994 a 55 f 930 f 4 " ;$ mobility="fixed"

User-Agent: IM-client/OMA1.0

Mercuro-Bronze/v4.0.1624.0

P-Preferred-Identity:

<sip:11111@open-ims.test>

Supported: path

P-Access-Network-Info: ADSL; eutran-

cell-id-3gpp $=00000000$

Privacy: none

Content-Length: 0

The $\mathrm{P}$ connects to the the $\mathrm{S}-\mathrm{CSCF}$, which in turn connects to the HSS, more exactly to the AuC part of it, using the Diameter protocol over the Cx interface, then gathers from there the AV - Authentication Vector string, which contains, as defined by AKA, 5 fields: RAND, AUTN, XRES, IK, CK. The RAND and AUTN values are then passed onto the SIP endpoint, as a nonce in the 401 Unauthorized message, WWW-Authenticate header, through the P, to the user. This message looks like this, where only the impacted fields are not collapsed, the capture being taken once the packet went out the P-CSCF's interface, and reached the SIP endpoint:

Status-Line: SIP/2.0

Unauthorized - Challenging the UE

Message Header

Via:

$\operatorname{SIP} / 2.0 / \mathrm{UDP}$

$172.20 .1 .1: 1143 ;$ rport $=1143 ;$ branch $=\mathrm{z} 9 \mathrm{hG} 4 \mathrm{~b}$ K1275411663890

From: <sip:11111@open-

ims.test>; tag $=6334$

SIP from address: sip:11111dopenims.test

SIP from address User Part: 11111 ims.test

SIP from address Host Part: open-

SIP tag: 6334

To:

$<$ sip: 11111 dopen-

ims . test $>$; tag=925746a962736b96138042b427

df 6549-2212

ims.test

SIP to address: sip:11111dopen-

SIP to address User Part: 11111

ims.test

SIP to address Host Part: open-

SIP

tag:

925746a962736b96138042b427df6549-2212

Call-ID:

50 a 5456166 f246b78f081ac2453ee4ea

CSeq: 901 REGISTER

Path:

$<$ sip:termepcscf.open-

ims.test: $4060 ; 1 r>$

Service-Route: <sip:origlscscf.open-

ims.test: $6060 ;$ lr>

Allow: INVITE, ACK, CANCEL, OPTIONS, BYE, REFER, SUBSCRIBE, NOTIFY, PUBLISH, MESSAGE, INFO

Server: Sip Express router (2.1.0dev1 OpenIMSCore (i386/linux))

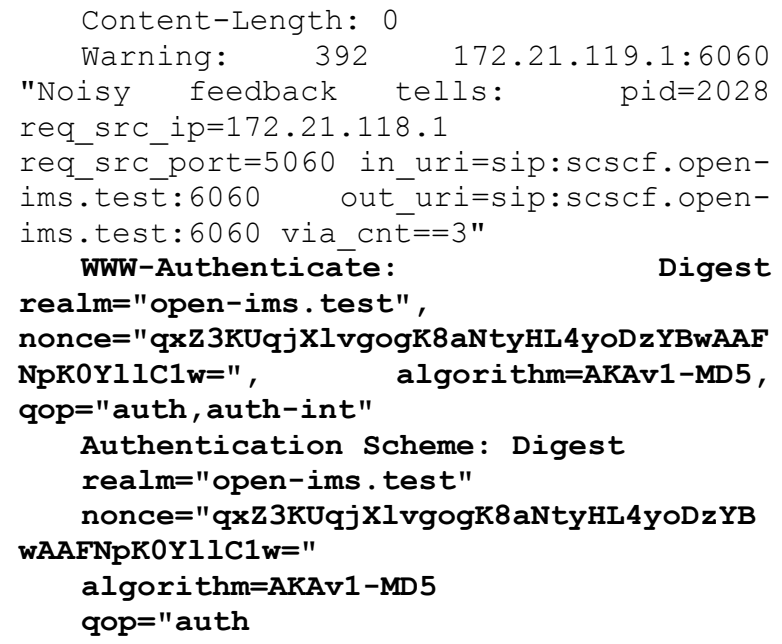

Using the AUTN information, the UE can authenticate the network. In its turn, the UE computes the RES - the response, and sends it back to the PCSCF in the new REGISTER message:

Request-Line: REGISTER sip:openims.test SIP/2.0

Message Header

Via:

$\mathrm{SIP} / 2.0 / \mathrm{UDP}$

172.20.1.1:1143; rport; branch=z9hG4bK1275 411663891

From:

ims.test $>$; tag $=6334$

To: <sip:11111@open-ims.test>

Call-ID:

M-

50 a 5456166 f246b78f081ac2453ee4ea

CSeq: 902 REGISTER

Max-Forwards: 70

Allow: INVITE, ACK, CANCEL, BYE, MESSAGE, OPTIONS, NOTIFY, PRACK, UPDATE, REFER

Contact:

<sip:11111@172.20.1.1:1143; transport=udp >; expires=600000; +deviceID="3ca50bcb-

$7 a 67-44 f 1-a f d 0-$

$994 a 55$ f930f 4 "; mobility="fixed"

User-Agent: IM-client/OMA1.0

Mercuro-Bronze/v4.0.1624.0

Authorization:

Digest

algorithm=AKAv1 -

MD5, username $=" 11111$ @open-

ims . test", realm="open-

ims . test", nonce="qxZ3KUqjXlvgogK8aNtyHL4

yoDzYBWAAFNpKOY11C1w=" , uri="sip : open-

ims . test", response=" 974679 fa1f988670b52e

bd3b058cf42a", qop=auth-in

P-Preferred-Identity:

<sip:11111dopen-ims.test>

Supported: path

P-Access-Network-Info:

ADSL; eutran-cell-id-3gpp $=00000000$

Privacy: none

Content-Length: 0

Now, the P-CSCF also authenticates the user, by verifying that the RES received from the US is the 
same as its XRES received via the S-CSCF, from the HSS. At the end of this, procedure, both the P-CSCF and the UE have the CK and IK keys. The P-CSCF sends a $200 \mathrm{OK}$ message to the UE, announcing the successful authentication:

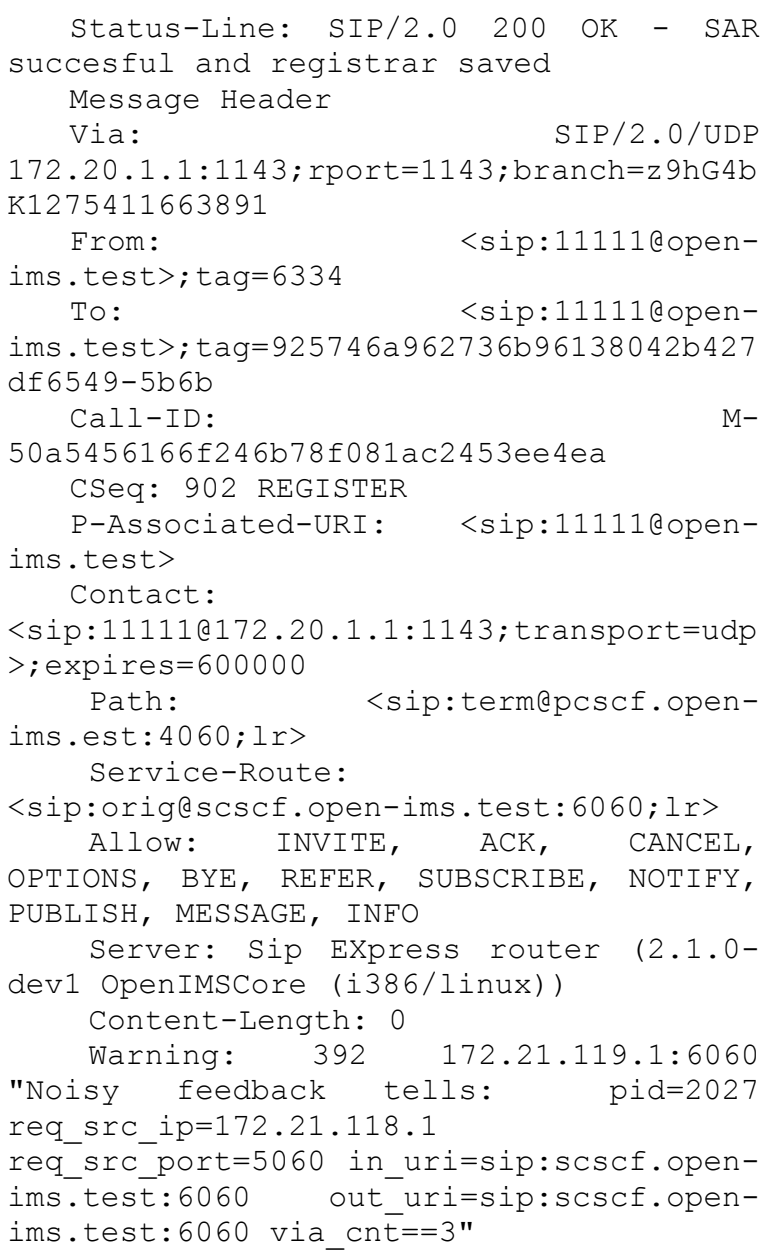

Following this procedure, the UE may use the $\mathrm{CK}$ and IK keys in order to create an end-to-end security tunnel between UICC and the P-CSCF, an IPSec tunnel. This tunnel may be statically or manually configured, provided that both UE and PCSCF have manually pre-defined IPSec methods, or by using IKEv1 or IKEv2 ISAKMP procedures in order to dynamically establish this tunnel.

\section{Proposed Authentication Model}

The mobile device has to follow a set of steps and has to meet a number of requirements in order to be authenticated to the $4 \mathrm{G}$ systems. Also, the $4 \mathrm{G}$ network is authenticated to the mobile device. The process is usually the $4 \mathrm{G}$ EPS-AKA procedure, sometimes also EAP-AKA or a special AKA procedure in cases where the UE follows a handover process from a $3 \mathrm{G}$ network to a $4 \mathrm{G}$ network. Due to its modularity characteristic, the IMS network also requires a separate authentication scheme in order for the mobile device to be able to access the IMS services provided by this network. The IMS scheme is AKA. Also, due to their modularity, the $4 \mathrm{G}$ and IMS networks are independent one of the other in terms of security access procedures. Nevertheless, the 4G and IMS networks are closely interoperating, because IMS provides services for the users that connect via the $4 \mathrm{G}$ network. Therefore, these two separate networks are connected to each-other. This way, the user that has followed all the security requirements to connect securely to the $4 \mathrm{G}$ network, now has to follow a set of steps and exchange SIP messages in order to authenticate to the IMS network. Looking at the entities that sit at the border of each of these two networks, the PGW in the $4 \mathrm{G}$ system and the P-CSCF in the IMS system, this paper proposes a security authentication model that has the purpose of leveraging the cryptographic and message exchange burden of the UE to IMS authentication process, by using one of the $4 \mathrm{G}$ entities closest to the IMS network as an authentication proxy. This entity is the PGW, which can have a proxy-authentication function from the UE perspective.

The PGW is the entity that is able to visualize the content of the eGTP control plane messages coming from the UE and it is also able to modify the content of these messages. This is its usual functionality. In the proposed design, the PGW has already established a secure communication to the UE. Then, at the moment of the UE's connection to the P-CSCF, the PGW is able to identify, parse and modify this message, in a manner that is transparent for the PCSCF. The model proposed in [18] should work but only if the IMS SIP/Diameter flow is also modified, because the UE sends the IMSI and the IMPI and the authentication data at once, and the GGSN directly forwards them to the P-CSCF. In the model proposed, the UE computes and sends its IMSI, IMPI and Authentication Data to the PGW, but the PGW does not send all this information to the P-CSCF at once.

Before the Initial Attach procedure, the UE selects ' $p$ ' and ' $g$ ' primes non-null and a secret ' $a$ ', then uses these values to derive value $\mathrm{A}$ from the Diffie-Hellman procedure: $A=g^{\wedge} a \bmod p$. Then the UE inserts value $\mathrm{A}, \mathrm{p}$ and $\mathrm{g}$ (or only $\mathrm{A}$ if $\mathrm{p}$ and $\mathrm{g}$ are somehow agreed upon from a pre-configured setting), then adds them to the PCO (Protocol Configuration Options) IE in the Attach Request message to the MME, PCO IE that propagates to the PGW in the Create Session Request message (step 1, Figure 5). This entity looks at the values received and computes value $B=g^{\wedge} b$ mod $p$, where $b$ is its key. After verifies that the UE is valid and sends it an IP (or an empty IP, if it is to be configured later dynamically), and includes also the B value in the PCO from the Create Session Response (step 2, Figure 5). The response gets back to the UE in the Attach Accept message. At this moment, the UE and the PGW derive a common Diffie-Hellman key $\mathrm{K}$, which they can use as a 
symmetrical encryption key or as a master key to derive further key for securing traffic between them. The UE sends the SIP Register (step 3, Figure 5) message and includes its secret key SIP-K, encrypted with $\mathrm{K}$. This message arrives at the PGW.

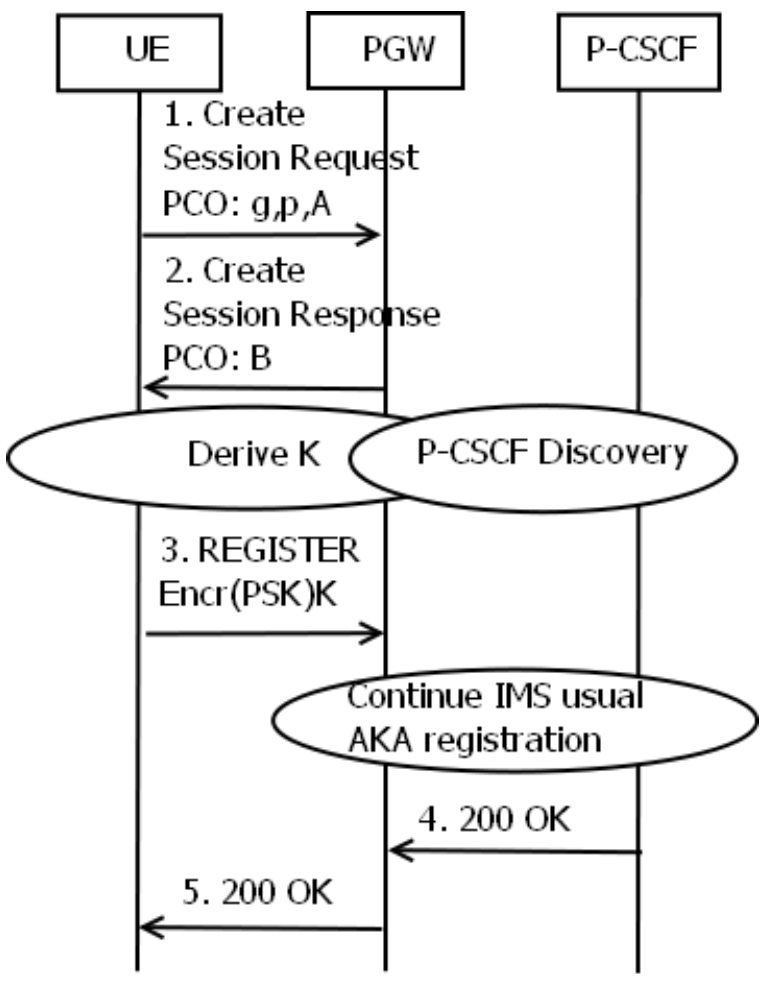

Figure 5. Proposed authentication procedure

The PGW then starts the P-CSCF discovery procedure and continues the IMS authentication procedure just as if it were the UE authenticating to the IMS. If the authentication is successful, then the P-CSCF forwards a 200 OK message (step 4, Figure 5) to the PGW and the PGW only forwards again this message to the UE, encapsulated in GTPv1-u header.

\subsection{Advantages and Disadvantages of this model}

This model has advantages, as well as disadvantages. The disadvantages of this model would be that the cryptographic computational for the UE increases initially, due to the Diffie-Hellman process. The UE has to do cryptographic computations anyway, but the encryption it is supposed to do for classic AKA is less intensive than the DH method. The upside here is that key derived from Diffie-Hellman can be used as a master key for any further secure key derivation procedures and this master key endures for as long as the UE is attached to the network. Another disadvantage is that this model induces a small change in the UE handset operation, in terms of SIP functionality. These changes are not great, meaning that the SIP message needs to have a header where to put the secret key, encrypted with the DH key. This disadvantage should not have a very high impact on the handset functionality, as the SIP protocol is highly flexible and there is no standard agreed upon SIP implementation for all the providers and terminals at this moment in time. So there is room for enhancements and additions.

Some of the advantages of this model are the following. The IMS system, being a relatively mature and stable one, does not have to change to implement this model. IMS was initially released by 3GPP as part of Release 5, in order to integrate this services network with the Release 5 UMTS technology. Since then, a lot of the IMS functionality has changed; even more, the various IMS implementations have diverged and converged, but there is no unified IMS implementation on all the networks nowadays. Even so, it is important to change as little as possible in a design that has already matured. By the model proposed, none of the IMS entities alters its functionality in order to accommodate the implementation of this proposed model. The P-CSCF discovery is the same, the PGW forwards the SIP Register message to the P-CSCF in the same manner, nothing is changed in that message by comparison with the Register message that the P-CSCF received with the classic IMS-AKA. The internal IMS AKA procedure remains unchanged. The 401 Unauthorized message that is sent by the P-CSCF to the UE is no longer forwarded to the UE, instead the PGW directly replies to this message: it verifies the AUTN, computes the RES and sends it to the P-CSCF. When receiving the $200 \mathrm{OK}$ from the $\mathrm{P}-\mathrm{CSCF}$, the PGW forwards this message to the UE and saves the status of the UE as properly registered to the IMS network.

Even though the model proposed is a bit slower in terms of computational speed on the mobile device and the PGW, the overall network load is reduced, by doing fewer exchanges over the network, therefore reducing the risk of packet loss and assuring a faster reply to the IMS system, directly from the PGW. As a collateral results of this model, the negotiation results in the derivation of a secure master key that can further be re-used for any other authentication or sensitive message exchange purposes between the UE and the PGW. This key does not have to be derived often, as the PGW is the attach anchor of the UE; so the only time when it would have to be re-computed is if the UE detaches. Also, the PGW may retain the IMS SIP-K key of the UE for further IMS reauthentication, which will happen much faster now. The PGW may retain the SIP-K key an re-use it even in the case when the UE may momentarily disconnect from the $4 \mathrm{G}$ network, then reconnect, even though this behavior is not necessarily desirable from a strong security perspective: in case where the UE disconnects from the $4 \mathrm{G}$ network, it should at first reestablish the DH process, and only then resend its IMS secret in a secure manner to the PGW. Also, the 
PGW may retain the IMS secret in cases where the UE only disconnects from the IMS network, but while it is still connected to the $4 \mathrm{G}$ network. This process should not lower the level of security.

Another aspect is the process of mobility. As long as the mobile device is located in the $4 \mathrm{G}$ access network, the PGW is the ultimate connectivity anchor point for the UE, its address does not change, unless the UE re-connects. Same thing is valid for the hybrid $3 \mathrm{G}-4 \mathrm{G}$ networks where the core network is $4 \mathrm{G}-\mathrm{EPC}$, because the PGW is the central entity there as well. If the access is non-3GPP, PGW continues to be the entity that handles the IP provisioning.

\section{Conclusions and Future work}

This paper presented the overall $4 \mathrm{G}$ and IMS architectures, using one of the scenarios most commonly encountered, where the UE is in the homenetwork. The UE authenticates to the $4 \mathrm{G}$ network, using MME as a proxy to the HSS. Then, when trying to access an application server, it may use the GAA architecture, either in GBA mode - using a shared secret located on the UICC and on the HSS, or using SSC - a PKI portal that assigns digital certificates to the UICC.

This paper also presented the classic IMS-SIPAKA procedure, an HTTP-like method for authenticating the SIP endpoint to the IMS network (HSS), via the CSCF servers available here. It presents as well a proposed authentication model for the IMS system, which is designed to provide faster and secure access to the IMS system, while relying on the security features of the $4 \mathrm{G}$ system, without producing relevant changes to the IMS core.

\section{References}

[1] RFC 3455 Private Header (P-Header) Extensions to the Session Initiation Protocol (SIP) for the 3rd-Generation Partnership Project (3GPP) -

http://www.ietf.org/rfc/rfc3455.txt (Access date: September, 2010)

[2] RFC 3329 Security Mechanism Agreement for the Session Initiation Protocol (SIP) http://www.faqs.org/rfcs/rfc3329.html (Access date: September, 2010)

[3] TS 33.401 - 3GPP SAE - Security architecture http://www.3gpp.org/ftp/Specs/archive/33_series/33.401/ (Access date: September, 2010)

[4] TS 33.402 - 3GPP SAE - Security aspects of non-3GPP accesses -

http://www.3gpp.org/ftp/Specs/archive/33_series/33.402/ (Access date: September, 2010)

[5] TS 33.203 - Access security for IP-based services http://www.3gpp.org/ftp/Specs/archive/33_series/33.203/ (Access date: September, 2010)
[6] TS 33.210 - Network Domain Security; IP network layer security http://www.3gpp.org/ftp/Specs/archive/33_series/33.210/ (Access date: September, 2010)

[7] TS 33.310 - Network Domain Security; Authentication Framework -

http://www.3gpp.org/ftp/Specs/archive/33_series/33.310/ (Access date: September, 2010)

[8] "IP Multimedia Subsystem (IMS) signaling core security" - Ivan Tirado Kennesaw State University, Kennesaw, GA, 2008 (Access date: September, 2010)

[9] "Security issues with the IP multimedia subsystem (IMS)" - Michael T. Hunter, Russell J. Clark, Frank S. Park - Georgia Institute of Technology, Atlanta, GA, 2007 (Access date: September, 2010)

[10] "Seamless mobility and standards" - David Binet France Telecom, Rennes - 2009 (Access date: September, 2010)

[11] Tech-Invite: http://tech-invite.com/ (Access date: August, 2010)

[12] TS 29.294 - Tunneling Protocol for Control plane (GTPv2-C)

http://www.3gpp.org/ftp/Specs/archive/29_series/29.274/

(Access date: September, 2010)

[13] TS 33.220 - Generic Authentication Architecture; Generic Bootstrapping Authentication http://www.3gpp.org/ftp/Specs/archive/33_series/33.220/ (Access date: September, 2010)

[14] TS $33.246 \quad-\quad$ Security of Multimedia Broadcast/Multicast Service http://www.3gpp.org/ftp/Specs/archive/33_series/33.246/ (Access date: August, 2010)

[15] TR 33.919 - Generic Authentication Architecture System Overview http://www.3gpp.org/ftp/Specs/archive/33_series/33.919/ (Access date: August, 2010)

[16] TS 33.221 - Support for Subscriber Certificates http://www.3gpp.org/ftp/Specs/archive/33_series/33.221/

(Access date: September, 2010)

[17] OpenIMSCore Project http://www.openimscore.org/ (Access date: September, 2010)

[18] "One-Pass Authentication and Key Agreement Procedure in IP Multimedia Subsystem for UMTS" Chung-Ming Huang and Jian-Wei Li

(Access date: February, 2011) 